\title{
Does Continuous Change Imply Continuity?
}

\author{
Sushil
}

Received: 9 July 2013/Accepted: 10 July 2013/Published online: 23 July 2013

(C) Global Institute of Flexible Systems Management 2013

The duality of continuity and change has conventionally been treated as a dilemma, i.e. either to be in continuity or to change. In the post modern concept of change management, this duality is reformulated as a paradox so as to deal with continuity and change side by side. In the context of strategic change, an interesting duality is of continuous change versus discontinuous change. Continuous change implies that the post change stage in an organization maintains a linkage with the pre change stage. For example, change happening in various business processes by way of continuous improvement leading to the evolution of the same process. In contrast, discontinuous change would mean almost no relationship between pre and post change stages. Some prominent examples of discontinuous change are: business process reengineering, and blue ocean strategy. Business process reengineering works on a clean slate approach leading to fundamental change in the process design resulting in dramatic improvement in performance. Blue ocean strategy deals with innovative offerings that create new market space making the competition irrelevant. In both the cases, there is hardly any linkage with the pre-change stage.

This poses a fundamental question that-does continuous change imply continuity? The answer to this may not be a crisp "Yes" or "No". It can be better formulated as a membership function on the continuum of continuity to change using fuzzy set theory. Traditionally, any type of change (whether continuous or discontinuous) has been termed as change, whereas continuity is defined as its polar

\section{Sushil ( $\square)$}

Department of Management Studies, Indian Institute of Technology Delhi, Vishwakarma Bhawan, Shaheed Jeet Singh Marg, New Delhi 110016, India

e-mail: jfsmspringer@gmail.com; profsushil@gmail.com opposite. It may better be conceptualized as a 'fuzzy set' having membership functions of continuity as well as change on this continuum.

The answer to the above question about "continuous change" would also depend, to a great extent, on how it is defined. What continuous change means to us? Does it mean the change that is happening in a continuous manner over a period of time, or it implies a linkage between pre and post change stages, or both? In both the definitions, there appears a stream of continuity in the happening of change. For example, a new born kid continuously grows and turn out to be an adult. Similarly, an entrepreneurial organization continuously expands and becomes a mega corporation. There are umpteen number of corporate examples of this kind such as that of Google, Microsoft, Apple, Infosys, and so on. Can we say that continuous process of change in these organizations resulting into major change (at times discontinuous) be treated as continuity of organization? At some stages, the rate of change might have been low, whereas in certain others it might have been quite high.

This brings out some other related questions. Do all types of continuous changes imply continuity? Can even a continuous change with very high rate of change may be treated as continuity? Does evolution happening continuously may at some stage turn out to be in metamorphosis? This can be seen in the natural evolution of biological systems resulting into creation of new species. This implies that continuous change may itself unfold into discontinuous change at some stage. For example, an organization like Cisco continuously acquiring other organizations may treat mergers and acquisitions as an ongoing activity and may form part of its continuity. But a large number of acquisitions, over a period of time, might change the basic identity of the organization. 
Another example of strategic change is diversification. Some organizations like GE keep on diversifying into new business areas and turn out to be conglomerates. In case of GE (previously known as General Electric), the main business of the company was in the area of electrical equipments. It kept on diversifying in a variety of areas such as medical equipments, financial products and so on, and at some stage the original business remained a minor proportion of the total turnover of the corporation. This almost transformed the character and identity of the organization and at some stage was renamed as GE. Similar trajectories can be found in other conglomerates as well. Another prominent example is of $3 \mathrm{M}$, which was previously dealing in mining and manufacturing and now offering a bouquet of innovative products. In this case, it is envisaged to generate nearly one-third of the turnover from the products developed in last 3 years. In such organizations, continuous change might emerge into discontinuous change in identity of the concerned organizations.
Thus, it would be difficult to draw a clear boundary line up to which the continuous change is about continuity and beyond that it is a major or radical change. This ambiguous nature of continuous and discontinuous change makes it a fuzzy set formulation and can be better managed as a continuous function in time and space. This discussion leads to the paradoxical behaviour that change implies continuity, and continuity implies change. Though, the two are seemingly opposite, they get intermingled with each other in an inseparable manner. The same can be said about continuous and discontinuous change. Continuous change may metamorphose into discontinuous change, and discontinuous change may create new processes of continuous change. For example, flowing stream strategy balances continuity and change in an enterprise, and blue ocean strategy creates a new market space that may convert later into a red ocean. 